\title{
Parameter determination and experimental verification of thermoelectric cooling for a low-temperature chemical reactor
}

\author{
WANG XiaoQun*, ZHAO ZhenLu, LIAO FangPing, WEI DiSheng \& DU ShanYi \\ School of Materials Science and Engineering, Beihang University, Beijing 100191, China
}

Received November 25, 2010; accepted December 20, 2010; published online April 13, 2011

\begin{abstract}
Thermoelectric coolers (TECs) were used to cool a low-temperature chemical reactor (LTR). A combined theoretical/ experimental study of the heat transfer in LTR with TECs was undertaken. First, two models of a TEC with a LTR junction were developed to evaluate the equilibrium and transient temperatures of the reactor inner wall. Next, two methods were used to determine the parameters of these models. Finally, the relationship between the transient temperatures of the reactor inner wall and cooling times was established. The results show that the calculated results of transient temperatures are in good agreement with the corresponding experimental data.
\end{abstract}

thermoelectric cooling, low temperature reactor, model parameter

Citation: Wang X Q, Zhao Z L, Liao F P, et al. Parameter determination and experimental verification of thermoelectric cooling for a low-temperature chemical reactor. Chinese Sci Bull, 2011, 56: 1611-1616, doi: 10.1007/s11434-011-4388-0

A thermoelectric cooler (TEC), also known as a Peltier cooler, is a semiconductor-based electronic component that functions as a small heat pump. When DC power is applied to a TE module, heat moves through the module from one side to the other proportional to the applied voltage. Notably, the thermoelectric module is only one element in the overall cooling system. Thermoelectric modules are solid state devices well-known to be reliable energy converters that are virtually maintenance-free. They are also noise-less and vibration-free as there are no mechanical moving parts. They are also smaller and lighter than comparable mechanical cooling systems. Their solid-state construction ensures high reliability, which is an advantage when used in systems that are not easily accessible after installation. For example, TECs used in air-conditioners offer significant advantages over more conventional cooling devices because they show better performance in active cooling, precise controllability, and reliability, as well as being environmental friendly, despite high costs because of low COP (Coefficient Of Performance) and production initialization

*Corresponding author (email: wangxiaoqun@buaa.edu.cn)
[1,2]. Phelan et al. [3] investigated a variety of refrigeration technologies, including thermoelectric, vapor compression, pulse tube, sorption and reverse Brayton, and found that only TECs are commercially available in small sizes. TECs are widely used to cool electronic devices [4-8] because of the plurality of advantages mentioned above. Comparative research on TECs has also been conducted. For instance, Xu and coworkers [10] have examined the heating process occurring in TECs, and obtained an empirical equation describing heat conduction with a heat source when the current density is evenly distributed along the direction of the $x$-axis.

In recent years, TECs have been applied in chemical industries or laboratories to cool small-volume parts [10-12]. For example, a TEC-based pre-concentrator [10,11] was developed for the laboratory to overcome the limitations of low-volume cryogenic devices employed in an automated rapid vapor concentrator. The operation of the thermoelectric trap have been examined and optimized with a system that utilized two independent gas chromatographs. TECs are also beginning to be used to cool high-temperature gascooled test reactors [12]. However, TECs are seldom used 
in low-temperature glass or metal reactors (as working temperatures are lower than room temperature) because their cooling capacity is limited. These types of low-temperature reactors are usually cooled by mechanical cooling systems. Cooling agents usually used are Freon, liquid nitrogen, liquid carbon dioxide, circulating oil or water. However, such mechanical cooling systems have several disadvantages. For example, these are incapable of controlling accurately the temperature of reactors, and their structures are complex and bulky, especially for cooling minireactors.

In this paper, we describe the use of TECs in the cooling of a mini-reactor (actual volume can range from a few to fifty milliliters), and study the temperature control of the reactor inner wall. Two models are developed to simulate the variation in temperature with cooling time and equilibrium temperatures of the reactor wall cooled by a TEC. Two methods to determine parameters of these models are compared. The influence of electric current on the performance of TEC systems is analyzed using these models. Finally, the results from simulations are compared with experimental data.

\section{System description}

In an exothermic chemical reaction, it is very important to keep the temperature of the reaction mixture at appropriate levels. The more accurately the temperature is controlled, the better experimental measurements can be repeated. For this reason, we consider here a typical situation where a commercial TEC is used to cool a mini-reactor designed by us in the laboratory to control the reaction temperature accurately. In addition, compared with conventional mechanical cooling systems, a TEC is silent, clean and simple to operate in situ. A heat sink with circulating water is used to dissipate heat from the hotter side of the TEC.

A schematic diagram of this system is depicted in Figure 1. The cooling system includes an alloy plate (of the same material as the chemical mini-reactor wall), a heating film (used to simulate the exothermal capacity of the chemical reaction expected), a TEC and a heat sink. The bonding of the TEC with the alloy wall on one side and the heat sink on the other is achieved by mechanical clamping with thermally-conductive grease between the contact surfaces. Most

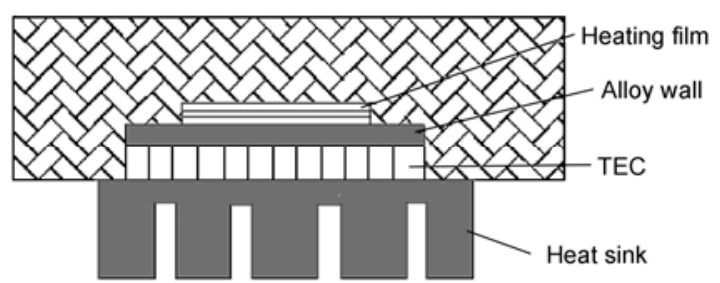

Figure 1 Schematic diagram of the cooling system used in the LTR. of the cooling system (including the alloy plate, the heating film, and the TEC) is insulated to reduce heat losses. In fact, in accordance with the inner volume of the mini-reactor and the expected exothermal capacity of the chemical reaction, the practical mini-reactor should need more than one of these cooling systems. One commercially-available TEC, TEC 12709 from Beijing Huimao Cooling Equipment Co, is $40 \mathrm{~mm}$ in width, $40 \mathrm{~mm}$ in length and $3.4 \mathrm{~mm}$ in thickness. When a direct current is applied, the TEC extracts heat from the wall of the mini-reactor to the heat sink. The heat sink dissipates the heat extracted by the TEC via the circulating water in the heat sink. The performance of the system was tested in a laboratory environment with tap water used as a coolant. Temperatures of the alloy wall and heat sink were measured with platinum resistance thermometers.

\section{Thermoelectric cooling system modeling}

\subsection{Steady state heat transfer model}

The operation of a thermoelectric module is based on the Peltier effect, which was discovered in 1834 . When direct current passes through a pair of $\mathrm{P}$ - and N-type semiconductor materials, one side of the junction is cooled and the other side is heated. Therefore, if the colder side of a TEC is attached to the outer side of a reactor alloy wall and the hotter side is connected to a heat sink, as shown in Figure 2, heat from the steel wall can be transferred through the TEC to the heat sink by connecting the device to a DC source. The heat sink passes heat to the circulating water.

It is assumed that all interfaces are in perfect thermal contact. In general, the thermal problem will be three dimensional. However, if the effective area of the TECs is nearly equal to the area of the alloy plate used as the reactor wall, it is reasonable to assume that the temperature varies only along a single dimension - the thickness of the TEC, as shown in Figure 2. This greatly simplifies to a 1D-thermoelectric problem.

The energy balance equations at the alloy wall and the heat sink are as follows [13]:

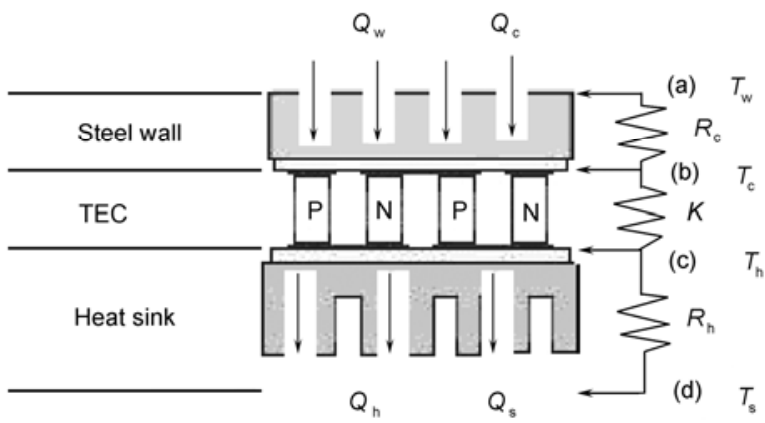

Figure 2 Steady-state model of a steel wall cooled by a TEC with heat sink. (a) Inner surface of steel wall; (b) joint between outer surface of steel wall and cold side of TECs; (c) hot side of TECs; (d) heat sink. 


$$
\begin{gathered}
Q_{\mathrm{w}}=Q_{\mathrm{c}}=\frac{T_{\mathrm{w}}-T_{\mathrm{c}}}{R_{\mathrm{c}}}, \\
Q_{\mathrm{c}}=\alpha I T_{\mathrm{c}}-\frac{1}{2} I^{2} R-K\left(T_{\mathrm{h}}-T_{\mathrm{c}}\right), \\
Q_{\mathrm{h}}=\alpha I T_{\mathrm{h}}+\frac{1}{2} I^{2} R-K\left(T_{\mathrm{h}}-T_{\mathrm{c}}\right), \\
Q_{\mathrm{s}}=Q_{\mathrm{h}}=\frac{T_{\mathrm{h}}-T_{\mathrm{s}}}{R_{\mathrm{h}}} .
\end{gathered}
$$

All material properties are assumed to be constant. When $T_{\mathrm{c}}$ and $T_{\mathrm{h}}$ are eliminated from eqs. (1)-(4), the respective heat balance equations at positions ' $a$ ' and 'd' in Figure 2 can be expressed as

$$
\begin{aligned}
Q_{\mathrm{w}}= & \alpha I T_{\mathrm{w}} \omega\left(1-\alpha I R_{\mathrm{h}}\right)-\frac{1}{2} I^{2} R \omega\left(1+2 K R_{\mathrm{h}}-\alpha I R_{\mathrm{h}}\right) \\
- & K\left(T_{\mathrm{s}}-T_{\mathrm{w}}\right) \omega, \\
Q_{\mathrm{s}}= & \alpha I T_{\mathrm{s}} \omega\left(1+\alpha I R_{\mathrm{c}}\right)+\frac{1}{2} I^{2} R \omega\left(1+2 K R_{\mathrm{c}}+\alpha I R_{\mathrm{c}}\right) \\
& -K\left(T_{\mathrm{s}}-T_{\mathrm{w}}\right) \omega,
\end{aligned}
$$

where

$$
\omega=\frac{1}{\left(\alpha I R_{\mathrm{c}}+1+K R_{\mathrm{c}}\right)\left(1-\alpha I R_{\mathrm{h}}\right)+\left(\alpha I R_{\mathrm{c}}+1\right) K R_{\mathrm{h}}} .
$$

If $R_{\mathrm{h}} \rightarrow 0$, eqs. (5)-(7) reduce to

$$
\begin{gathered}
Q_{\mathrm{c}}=\alpha I T_{\mathrm{c}} \omega-\frac{1}{2} I^{2} R \omega-K\left(T_{\mathrm{h}}-T_{\mathrm{c}}\right) \omega, \\
Q_{\mathrm{h}}=\alpha I T_{\mathrm{h}} \omega\left(1+\alpha I R_{\mathrm{c}}\right)+\frac{1}{2} I^{2} R \omega\left(1+2 K R_{\mathrm{c}}+\alpha I R_{\mathrm{c}}\right) \\
-K\left(T_{\mathrm{h}}-T_{\mathrm{c}}\right) \omega,
\end{gathered}
$$

where

$$
\omega=\frac{1}{\alpha I R_{\mathrm{c}}+1+K R_{\mathrm{c}}} .
$$

When heat is transferred from the reactor mixture to the steel wall $Q_{\mathrm{w}}$, the temperature $T_{\mathrm{h}}$ of the hotter TEC side and the TEC parameters, including the Seebeck coefficient $\alpha$, electrical resistance $R$ and thermal conductivity $K$, are all given. The temperature $T_{\mathrm{w}}$ of the inner surface (directly in contact with the reaction mixture) of the alloy wall attached to the colder TEC side can be estimated using the following equation

$$
\begin{aligned}
T_{\mathrm{w}}= & \frac{\frac{Q_{\mathrm{w}}}{\omega}+\frac{1}{2} I^{2} R+K T_{\mathrm{h}}}{\alpha I+K} \\
= & \frac{Q_{\mathrm{w}}\left(1+\alpha I R_{\mathrm{c}}+K R_{\mathrm{c}}\right)+\frac{1}{2} I^{2} R+K T_{\mathrm{h}}}{\alpha I+K} .
\end{aligned}
$$

\subsection{Transient heat transfer model}

In the transient state, the problem is much more complex than for the steady state just presented. The cooling capacity of the TEC is related to $T_{\mathrm{h}}$ although all TEC parameters (viz. $\alpha, R$ and $K)$ are considered here as a constant independent of time $t$. The physical model for the transient state is shown in Figure 3.

The energy balance of the system is then

$$
Q_{\text {input }}=Q_{\text {output }}+\Delta H_{\text {system }},
$$

where $Q_{\text {input }}$ is the heat transferred from the inside of the reactor to a TEC via the steel wall, $Q_{\text {output }}$ is the heat transferred to the TEC from the alloy wall, and $\Delta H_{\text {system }}$ is the increase in energy of the alloy wall. At any time $t, \Delta H_{\text {system }}$ is given by

$$
\Delta H_{\text {system }}=\rho c V \frac{\mathrm{d} T_{\mathrm{w}}}{\mathrm{d} t}=\rho c S D \frac{\mathrm{d} T_{\mathrm{w}}}{\mathrm{d} t},
$$

where $c$ and $\rho$ are the specific thermal capacity and density respectively of the alloy wall, and $S$ the area of the alloy wall or of the electric module. Because the alloy wall is thin $(D=3 \mathrm{~mm})$ and the thermal conductivity of the alloy wall is high $(\lambda=169 \mathrm{~W} / \mathrm{m} \mathrm{K})$, it can be assumed that there is a small temperature difference $\left(\Delta T_{\mathrm{w}}=\frac{Q_{\mathrm{w}} D}{\lambda}=0.09 \mathrm{~K}\right)$ between the inner surface and the outer surface of the alloy wall although the heat flux is very large $\left(Q_{\mathrm{w}}=5000 \mathrm{~W} / \mathrm{m}^{2}\right)$ through the alloy wall. As a result, it can be assumed that the temperature of the alloy wall $T_{\mathrm{w}}$ along the thickness dimension is constant independent of time $t$. Thus, eq. (12) can be expressed as

$$
Q_{\text {input }}=Q_{\text {output }}+\rho c S D \frac{\mathrm{d} T_{\mathrm{w}}}{\mathrm{d} t} .
$$

It is also assumed that the interface between the alloy wall and the colder TEC side is in perfect thermal contact. As a result, the temperature of the colder TEC side is equal to that of the alloy wall, $T_{\mathrm{c}}=T_{\mathrm{w}}$. The heat absorbed by the TEC may be calculated using eq. (2), thereby modifying eq. (14) to

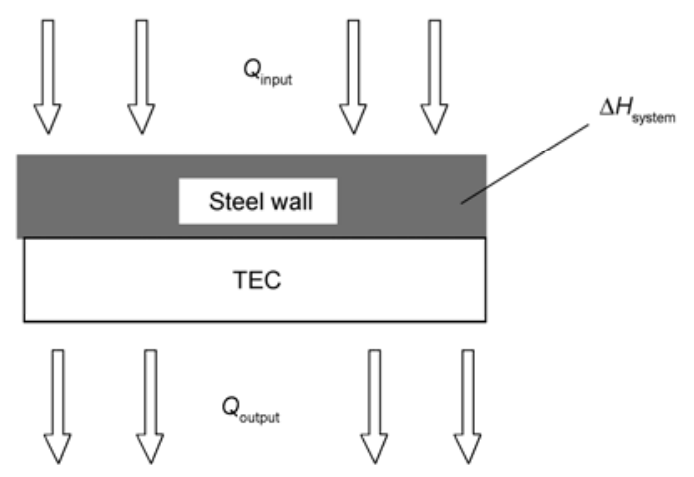

Figure 3 Transient model of heat being transferred from the reactor to the heat sink. 


$$
Q_{\text {input }}=\alpha I T_{\mathrm{w}}-\frac{1}{2} I^{2} R-K\left(T_{\mathrm{h}}-T_{\mathrm{w}}\right)+\rho c S D \frac{\mathrm{d} T}{\mathrm{~d} t} .
$$

It is further assumed that for all times $t$, the heat flux transferred from the reactor is constant, and the interface between the hotter TEC side and the heat sink is in perfect thermal contact $\left(T_{\mathrm{h}}=T_{\mathrm{s}}\right)$. Thus, eq. (15) is expressed as

$$
Q_{\mathrm{w}}-\left[\alpha I T_{\mathrm{w}}-\frac{1}{2} I^{2} R-K\left(T_{\mathrm{s}}-T_{w}\right)\right]=\rho c S D \frac{\mathrm{d} T_{\mathrm{w}}}{\mathrm{d} t} .
$$

The initial temperature of the alloy wall is stipulated to be

$$
T_{\mathrm{w}}=T_{\mathrm{o}}, t=0 .
$$

The temperature of the steel wall is calculated using the following equation:

$$
T_{\mathrm{w}}=\mathrm{e}^{-\frac{\alpha I+K}{\rho c S D}(t+A)}+\frac{\frac{1}{2} I^{2} R+K T_{\mathrm{s}}+Q_{\mathrm{w}}}{\alpha I+K},
$$

where $A$ is a constant that can be determined according to the initial condition $t=0, T_{\mathrm{w}}=T_{\mathrm{o}}$.

\subsection{Parameters of the models}

Two methods are considered to determine the parameters of the proposed models mentioned above. One is to calculate the parameters from average temperature $T_{\mathrm{m}}$; the other is to extract the parameters from the manufacturer's data of TECs [14].

Method I

Currently, commercial TEC devices are made from semiconducting materials, such as $\mathrm{Bi}_{2} \mathrm{Te}_{3}$. According to test results by Melcor Corp., the relationship between the Seebeck coefficient $(\alpha)$, electrical resistance $(R)$, thermal conductivity $(K)$ and average temperature $\left(T_{\mathrm{m}}\right)$ can be described as

$$
\begin{gathered}
\alpha_{\mathrm{P}}=-\alpha_{\mathrm{N}}=\left(22224.0+930.6 T_{\mathrm{m}}-0.9905 T_{\mathrm{m}}{ }^{2}\right) \times 10^{-9} \mathrm{~V} / \mathrm{K},(19) \\
r_{\mathrm{P}}=r_{\mathrm{N}}=\left(5112.0+163.4 T_{\mathrm{m}}+0.6279 T_{\mathrm{m}}{ }^{2}\right) \times 10^{-10} \Omega / \mathrm{m}, \quad(20) \\
k_{\mathrm{P}}=k_{\mathrm{N}}=\left(62605.0-277.7 T_{\mathrm{m}}+0.4131 T_{\mathrm{m}}{ }^{2}\right) \times 10^{-4} \mathrm{~W} /(\mathrm{m} \mathrm{K}),(21)
\end{gathered}
$$

where $T_{\mathrm{m}}$ is the average of temperatures $T_{\mathrm{h}}$ and $T_{\mathrm{w}}$. Subscripts $\mathrm{P}$ and $\mathrm{N}$ signify $\mathrm{P}$ and $\mathrm{N}$-type arms, respectively.

A TEC consists of a number of $\mathrm{P}$ - and N-type semiconductor pairs (couples) connected electrically in series and sandwiched between two ceramic plates. We can then calculate the Seebeck coefficient $\alpha$, electrical resistance $R$, and thermal conductivity $K$ of a TEC using the following equations:

$$
\begin{gathered}
\alpha=2 N \alpha_{\mathrm{p}}, \\
R=2 N \rho_{p} \frac{l}{s},
\end{gathered}
$$

$$
K=2 N k_{p} \frac{s}{l},
$$

where $N$ is the number of couples, $l$ is the length of the $\mathrm{P}$ and $\mathrm{N}$ arms, $s$ is the cross-sectional area of the $\mathrm{P}$ and $\mathrm{N}$ arms.

Method II

The parameters of the proposed models can be extracted from the manufacturer's data of a TEC [14] as

$$
\begin{gathered}
\alpha=\frac{U_{\text {max }}}{T_{h}}, \\
R=\frac{U_{\text {max }}}{I_{\text {max }}} \times \frac{T_{\mathrm{h}}-\Delta T_{\text {max }}}{T_{\mathrm{h}}}, \\
K=\frac{U_{\text {max }} \times I_{\text {max }}}{2 \times \Delta T_{\text {max }}} \times \frac{T_{\mathrm{h}}-\Delta T_{\text {max }}}{T_{\mathrm{h}}},
\end{gathered}
$$

where $\Delta T_{\max }$ is the largest temperature differential that can be obtained between the hot and cold ceramic plates of a TEC for a given level of $T_{\mathrm{h}}, I_{\max }$ is the input current which will produce the maximum possible $\Delta T$ across a TEC, and $U_{\max }$ is the DC voltage that delivers the maximum possible $\Delta T$ at the supplied $I_{\max }$.

\section{Results of simulation and experiment}

A commercial thermoelectric cooler TEC12709 is tested over a broad range of working conditions. All tests were made under low electrical currents $(I=1-9$ A) because performance is better at lower currents. The tests were also performed with heat transfers $Q_{\mathrm{c}}=Q_{\mathrm{w}}=10,20,30$ or $40 \mathrm{~W}$.

\subsection{Steady-state analysis}

In accordance with Method I, we start with the number of couples $N=127$ and average temperature $T_{\mathrm{m}}=300 \mathrm{~K}$. For a $\mathrm{PN}$-pair, the length of the $\mathrm{P}$ and $\mathrm{N}$ arms is $l=1 \mathrm{~mm}$, giving area $s=1.37 \times 1.37 \mathrm{~mm}^{2}$. Applying eqs. (19)-(24), one can calculate the model parameters to obtain values: $\alpha=0.0540$ $\mathrm{V} / \mathrm{K}, R=1.50 \Omega, K=0.785 \mathrm{~K} / \mathrm{W}$.

Similarly, for Method II, we find from the manufacturer's data sheets: under $T_{\mathrm{h}}=300 \mathrm{~K}$ condition $\Delta T_{\max }=62 \mathrm{~K}$, $I_{\max }=9 \mathrm{~A}, U_{\max }=15.2 \mathrm{~V}$, and $Q_{\max }=89.2 \mathrm{~W}$. Applying eqs. (25)-(27), one can calculate the model parameters: $\alpha=0.0507 \mathrm{~V} / \mathrm{K}, R=1.34 \Omega, K=0.875 \mathrm{~K} / \mathrm{W}$.

Equilibrium temperatures of the reactor wall $T_{\mathrm{w}}$, can be calculated from eqs. (10) and (11). Here, $T_{\mathrm{h}}=300 \mathrm{~K}$, the input current $I$ ranges from 1 to $9 \mathrm{~A}, R_{\mathrm{c}}=D / \lambda S=1.1 \times 10^{-2}$ $\mathrm{K} / \mathrm{W}$, and $Q_{\mathrm{w}}=10,20,30$ or $40 \mathrm{~W}$.

Figure 4 shows the results of calculations using the steadystate model for the TEC system and the experimental data for $Q_{\mathrm{c}}=10,20,30,40 \mathrm{~W}$. In this figure, the dashed line is the calculation result using model parameters determined by Method I, the solid line is the calculation result with model parameters 
obtained by Method II, and points represent data from experimental measurements. As one can see, results from Method II are in good agreement with the experimental data under different $Q_{\mathrm{c}}$. Thus, Method II is better in determining these parameters than Method I. From Figure 4, it is also clear that the equilibrium temperature is lowest when the input current $I$ is set at 6 A given any set cooling capacity.

\subsection{Transient analysis}

Transient temperatures of the reactor wall are calculated using eqs. (17) and (18). The temperature of the heat sink is measured as $T_{\mathrm{s}}=300 \mathrm{~K}$; the physical properties of alloy wall are $c=881 \mathrm{~J} /(\mathrm{kg} \mathrm{K}), \rho=2700 \mathrm{~kg} / \mathrm{m}^{3}, S=4 \mathrm{~cm} \times 4 \mathrm{~cm}$, and $D=3 \mathrm{~mm}$.
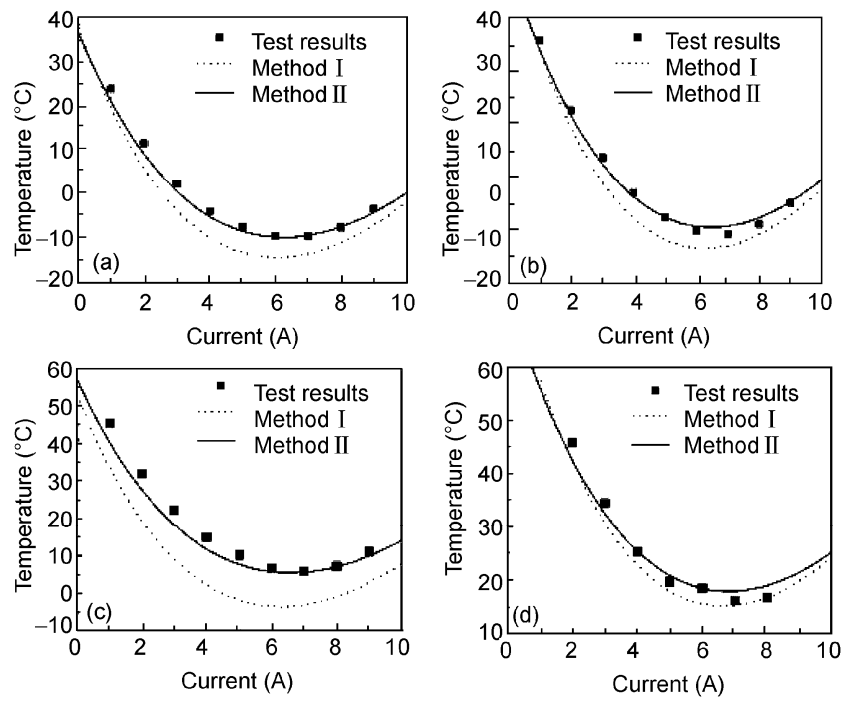

Figure 4 Calculational results of the steady-state model and test data under different $Q_{\mathrm{c}}$ conditions. (a) $Q_{\mathrm{c}}=10 \mathrm{~W}$; (b) $Q_{\mathrm{c}}=20 \mathrm{~W}$; (c) $\mathrm{Q}_{\mathrm{c}}=30 \mathrm{~W}$; (d) $Q_{\mathrm{c}}=40 \mathrm{~W}$.
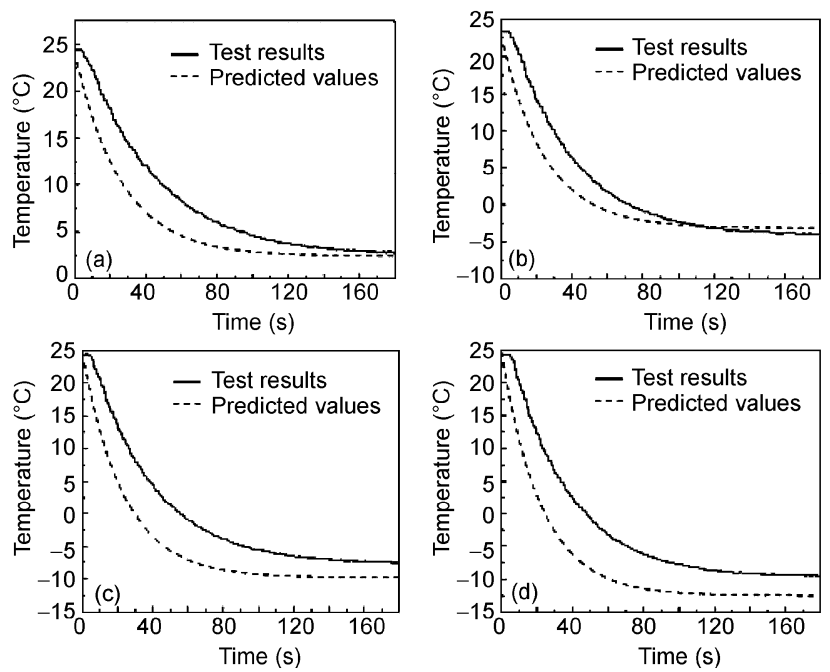

Figure 5 Calculation results of the transient model and test data at different current $\left(Q_{\mathrm{c}}=10 \mathrm{~W}\right)$. (a) Current $3 \mathrm{~A}$; (b) current $4 \mathrm{~A}$; (c) current $5 \mathrm{~A}$; (d) current $6 \mathrm{~A}$.
In this study, different TEC input currents at a pre-determined cooling capacity of $10 \mathrm{~W}$ are considered. The time- dependence of the temperature at the inner surface of the alloy wall is presented in Figure 5. Here, the dashed line is the calculated result with the model parameters determined by Method II, while the solid line corresponds to the experimental results. Clearly the calculated curve is in good agreement with the experimental curve, although the rate of decrease in the measured temperature of the alloy wall with cooling time is slightly lower than the calculated results. The difference seems to be more apparent at higher electrical currents. The reason may be due to the fact that thermal resistances are ignored between the TEC and the alloy wall and between the TEC and the heat sink.

\section{Conclusions}

The equilibrium wall temperatures of an LTR cooled by a TEC and temperature changes with applied direct current were well-described by the steady-state model. The transient temperatures and their change with cooling time were calculated using the transient model. In particular, two methods were used to determine the model parameters. It was found better to extract the parameters of the proposed models from the manufacturer's data of the TEC than from the average temperature $T_{\mathrm{m}}$. The results of calculations using the steady-state model were in good agreement with the experimental data at different values of $Q_{\mathrm{c}}$. The equilibrium temperature was lowest when the input current $I$ was $6 \mathrm{~A}$ at any of the pre-set cooling capacities. A good fit was also achieved between the experimental and simulation results of the transient model although the rate of decrease in measured temperatures is a little slower than estimated results.

Overall, we revealed that not only can TECs be used in the cooling of mini-reactors, but the steady-state and transient models can also be used to aid the design of practical LTRs.

The authors would like to thank Dr. Huang Chen, Xu Jun and Zhu Xiaoguang for their assistance in the research work.

\section{Nomenclature}

A, constant determined by eqs. (17) and (18);

c, thermal capacity ratio of the alloy wall;

D, thickness of the alloy wall;

$\Delta H_{\text {system }}$ energy increase for the alloy wall with increasing of the temperature;

I, current through the thermoelectric module;

$I_{\max }$ input current which will produce the maximum possible $\Delta T$ across a TEC;

$k$, thermal conductivity of $\mathrm{P}$ and $\mathrm{N}$-type arms;

$K, \quad$ total thermal conductivity of thermoelectric module;

$l$, length of P-type and $\mathrm{N}$-type arms; 
$N$ number of PN semiconductor couples;

$Q_{\mathrm{c}}, \quad$ cooling capacity in the thermoelectric cooling system;

$Q_{\mathrm{h}}, \quad$ heat dissipated from hot-side of the thermoelectric module;

$Q_{\text {input }} \quad$ heat transfer from the inside of the reactor to a TEC through the

alloy wall;

$Q_{\text {output }}, \quad$ heat transfer to the TEC from the alloy wall;

$Q_{\text {s }}, \quad$ heat dissipated by circulating water in the heat sink;

$Q_{\mathrm{w}}, \quad$ heat dissipated by the reactor mixture into the alloy wall;

$r, \quad$ electrical resistance of P-type and N-type arms;

$R, \quad$ total electrical resistance of thermoelectric module;

$R_{\mathrm{c}} \quad$ total thermal resistance of the alloy wall and the cold side of a TEC;

$R_{\mathrm{b}}$, total thermal resistance of the hot-side of the thermoelectric

$R_{\mathrm{h}}, \quad$ module and the heat sink;

$s, \quad$ area of the one $\mathrm{P}$ or $\mathrm{N}$-type arm;

$S, \quad$ area of the alloy wall or area of the electric module;

$t$ time;

$T_{\mathrm{c}}, \quad$ cold side temperature of thermoelectric module;

$T_{\mathrm{h}}$, hot-side temperature of the thermoelectric module;

$T_{\mathrm{m}}, \quad$ average temperature between cold and hot sided of a TEC;

$T_{\mathrm{s}}$, temperature of the heat sink;

$\Delta T_{\max }, \quad$ largest temperature differential between the hot and cold ceramic plates of a TEC

$T_{\mathrm{w}}, \quad$ temperature of the alloy reactor wall;

$\Delta T_{\mathrm{w}}, \quad$ temperature difference between the outside and inside of the alloy wall;

$U_{\max }, \quad$ DC voltage that delivers the maximum possible $\Delta T$ at the supplied $I_{\max }$

$V$ volume of the alloy wall;

Greek symbols;

$\alpha, \quad$ Seebeck coefficient of thermoelectric module;

$\rho, \quad$ density of the alloy wall;

$\lambda, \quad$ thermal conductivity of the alloy wall; $\omega$

$$
\omega=\frac{1}{\left(\alpha I R_{c}+1+K R_{c}\right)\left(1-\alpha I R_{h}\right)+\left(\alpha I R_{c}+1\right) K R_{h}} .
$$

1 Riffat S B, Qiu G Q. Comparative investigation of thermoelectric air-conditioners versus vapour compression and absorption airconditioners, Appl Thermal Eng, 2004, 24:1979-1993

2 Gao M, Rowe D M. Experimental evaluation of prototype thermoelectric domestic-refrigerators, Appl Energy, 2006, 83: 133-152

3 Patrick E P, Victor A C, Yu T, et al. Current and future miniature refrigeration cooling technologies for high power microelectronics. IEEE Trans Comp Pack Tech, 2002, 25: 356-365

4 Simons R E, Chu R C. Application of thermoelectric cooling to electronic equipment: A review and analysis. In: Sixteenth IEEE SEMITHERM Symposium, March 21-23, 2000, 1-9

5 DiSalvo, Francis J. Thermoelectric cooling and power generation. Science, 1999, 285: 703-706

6 Fukutani K, Shakouri A. Design of bulk thermoelectric modules for integrated circuit thermal management. IEEE Trans Comp Packaging Tech, 2006, 29: 750-757

7 Yamanashi M. A new approach to optimum design in thermoelectric cooling systems. J Appl Phys, 1996, 80: 5494-5502

8 Zhu D S, Lei J X, Wang C H, et al. Research progress in electronic comonent heat dissipation using thermoelectr ic cooling technology. Microelectronics, 2009, 39: 94-100

$9 \mathrm{Xu} \mathrm{S} \mathrm{L,} \mathrm{Yu} \mathrm{G} \mathrm{L,} \mathrm{He} \mathrm{D,} \mathrm{Derivation} \mathrm{of} \mathrm{new} \mathrm{formulae} \mathrm{of} \mathrm{thermoelectric}$ cooling. Chinese Sci Bull, 1992, 37: 1868-1868

10 Gehrke M, Kapila S, Hambacker K, et al. Design of an automated rapid vapor concentrator and its application in nitroaromatic vapor sampling. Proceeding of SPIE, SPIE Digital Library, 2000

11 Hambacker K, Kapila S, Nam P, et al. A rapid vapor concentrator and detection system for nitroaromatics. Proceeding of SPIE, 2001

12 Ishiyama S, Fukaya K, Eto M. Synthesis and fabrication technique of $\mathrm{SiC} / \mathrm{SiC}$ thermo elements. Key Eng Mater, 1999, 159-160: 423

13 Ioffe A F. Semiconductor thermoelement and thermoelectric cooling. Infosearch, 1957

14 Simon L, Sam B Y. Analysis of thermoelectric coolers by a spicecompatible equivalent-circuit model. IEEE Power Electro Lett, 2005, 3: $63-66$

Open Access This article is distributed under the terms of the Creative Commons Attribution License which permits any use, distribution, and reproduction in any medium, provided the original author(s) and source are credited. 